\title{
Oscillatory Noncollinear Magnetism Induced by Interfacial Charge Transfer in Superlattices Composed of Metallic Oxides
}

\author{
Jason D. Hoffman, ${ }^{1, *}$ Brian J. Kirby, ${ }^{2}$ Jihwan Kwon, ${ }^{3}$ Gilberto Fabbris, ${ }^{4}$ D. Meyers, ${ }^{4}$ John W. Freeland, ${ }^{5}$ \\ Ivar Martin, ${ }^{1}$ Olle G. Heinonen, ${ }^{1}$ Paul Steadman, ${ }^{6}$ Hua Zhou, ${ }^{5}$ Christian M. Schlepütz, ${ }^{5}$ Mark P. M. Dean, ${ }^{4}$ \\ Suzanne G. E. te Velthuis, ${ }^{1}$ Jian-Min Zuo, ${ }^{3}$ and Anand Bhattacharya ${ }^{1,7, \dagger}$ \\ ${ }^{1}$ Materials Science Division, Argonne National Laboratory, Argonne, Illinois 60439, USA \\ ${ }^{2}$ NIST Center for Neutron Research, National Institute of Standards and Technology, \\ Gaithersburg, Maryland 20899, USA \\ ${ }^{3}$ Department of Materials Science and Engineering, University of Illinois at Urbana-Champaign, \\ Urbana, Illinois 61801, USA \\ ${ }^{4}$ Department of Condensed Matter Physics and Materials Science, \\ Brookhaven National Laboratory, Upton, New York 11973, USA \\ ${ }^{5}$ Advanced Photon Source, Argonne National Laboratory, Argonne, Illinois 60439, USA \\ ${ }^{6}$ Diamond Light Source, Diamond House, Harwell Science and Innovation Campus, \\ Didcot, Oxfordshire OX11 ODE, United Kingdom \\ ${ }^{7}$ Nanoscience and Technology Division, Argonne National Laboratory, Argonne, Illinois 60439, USA \\ (Received 16 October 2015; revised manuscript received 15 June 2016; published 22 November 2016) \\ Interfaces between correlated complex oxides are promising avenues to realize new forms of magnetism \\ that arise as a result of charge transfer, proximity effects, and locally broken symmetries. We report on \\ the discovery of a noncollinear magnetic structure in superlattices of the ferromagnetic metallic oxide \\ $\mathrm{La}_{2 / 3} \mathrm{Sr}_{1 / 3} \mathrm{MnO}_{3}$ (LSMO) and the correlated metal $\mathrm{LaNiO}_{3}$ (LNO). The exchange interaction between \\ LSMO layers is mediated by the intervening LNO, such that the angle between the magnetization of \\ neighboring LSMO layers varies in an oscillatory manner with the thickness of the LNO layer. The \\ magnetic field, temperature, and spacer thickness dependence of the noncollinear structure are inconsistent \\ with the bilinear and biquadratic interactions that are used to model the magnetic structure in conventional \\ metallic multilayers. A model that couples the LSMO layers to a helical spin state within the LNO fits the \\ observed behavior. We propose that the spin-helix results from the interaction between a spatially varying \\ spin susceptibility within the LNO and interfacial charge transfer that creates localized $\mathrm{Ni}^{2+}$ states. Our \\ work suggests a new approach to engineering noncollinear spin textures in metallic oxide heterostructures. \\ DOI: 10.1103/PhysRevX.6.041038 \\ Subject Areas: Condensed Matter Physics, Magnetism, \\ Materials Science
}

Oxide interfaces have attracted considerable interest in recent years, as the reconstruction of charge, orbital, and spin states on the nanometer scale gives rise to novel phenomena that range from interfacial superconductivity to multiferroic behavior [1]. In this context, interfaces between oxides that are metallic in the bulk are particularly intriguing, as the large electronic compressibility, the relatively large bare dielectric constant, and band misalignment can work in concert to

\footnotetext{
*jasonhoffman@fas.harvard.edu;
}

Present address: Department of Physics, Harvard University, Cambridge, MA 02138, USA and Department of Physics and Astronomy, University of British Columbia, Vancouver, BC V6T 1Z4, Canada.

† anand@anl.gov

Published by the American Physical Society under the terms of the Creative Commons Attribution 3.0 License. Further distribution of this work must maintain attribution to the author(s) and the published article's title, journal citation, and DOI. create significant interfacial charge transfer over a region of several unit cells [2-4]. In oxides derived from correlated Mott insulators, this effect can manifest latent electronic and magnetic instabilities, leading to new collective states near the interface.

While a large body of work has emerged on heterostructures that incorporate insulating complex oxides [5-16], those created exclusively with metallic oxide constituents have been far less explored [17-19], despite the technological importance and wide range of behaviors observed in multilayers of conventional metals. The discovery of giant magnetoresistance (GMR) [20,21] and the subsequent demonstration of a tunable collinear exchange coupling in such structures [22] opened new pathways to high-density magnetic data storage. Multilayers with noncollinear magnetic ordering, however, are rarer, as such structures require a delicate balance between exchange energies, which only occurs under special circumstances [23-27]. Engineering robust noncollinear magnetic states at oxide interfaces presents new opportunities to explore novel effects, such 
as spin-transfer torque [28], long-range superconducting proximity effects [29], multiferroicity [30-32], magnetic Skyrmion phases [33,34], and new phenomena that emerge from correlated electronic states not found in conventional metals.

In this work, we show that charge transfer at the interface between two widely studied correlated metallic oxides $\mathrm{La}_{2 / 3} \mathrm{Sr}_{1 / 3} \mathrm{MnO}_{3}$ (LSMO) and $\mathrm{LaNiO}_{3}$ (LNO) can stabilize a novel noncollinear magnetic structure. In the bulk, LSMO is a ferromagnetic half-metal at low temperatures. LNO is a correlated paramagnetic metal, where epitaxial strain, dimensional confinement, and interfacial exchange interactions are known to stabilize long-range charge and magnetic ordering and correlated insulating states in thin films and heterostructures [9-11,13,35-39]. Using polarized neutron reflectometry, we find that an intrinsically noncollinear magnetic structure develops in superlattices of LSMO and LNO, grown with oxide molecular beam epitaxy (MBE). The magnitude of noncollinearity (the angle between the magnetization of adjacent LSMO layers) oscillates with LNO thickness, without ever becoming antiferromagnetic (i.e., an angle of $180^{\circ}$ ). The magnetic field, temperature, and LNO-layer thickness dependence of the exchange coupling between the LSMO layers is incompatible with a model based on the combination of bilinear and biquadratic coupling that is widely used to characterize noncollinear magnetic interactions in conventional metallic heterostructures. Rather, we show that the observed behavior is consistent with the development of a proper-screw-type magnetic order within LNO with a period of 5-7 unit cells along the (001) propagation direction. This structure persists to near ambient temperatures, above the magnetic ordering temperature known for any of the rare-earth nickelates. The helical spin structure proposed here is believed to result from a coupling between a momentum-dependent spin susceptibility $\chi(q)$ within the LNO layers and localized $\mathrm{Ni}^{2+}$ spins produced by charge transfer at the LNO/LSMO interface, which we measure using x-ray spectroscopy.

Epitaxial superlattices of LSMO and LNO were coherently grown on (001)-oriented $\mathrm{SrTiO}_{3}$ (STO) and $\left(\mathrm{LaAlO}_{3}\right)_{0.3^{-}}\left(\mathrm{Sr}_{2} \mathrm{TaAlO}_{6}\right)_{0.7}$ (LSAT) substrates at $600{ }^{\circ} \mathrm{C}$ using oxide MBE. The number of repeats, which each consists of nine unit cells of LSMO $(c=0.387 \mathrm{~nm})$ and $n$ unit cells of LNO $(c=0.382 \mathrm{~nm})$, where $1 \leq n \leq 9$, was adjusted to achieve a total thickness of about $60 \mathrm{~nm}$ (about 160 unit cells). The growth was monitored in situ by reflection high-energy electron diffraction (RHEED), and the maxima in the oscillating specular spot intensity correspond to the deposition of a single unit-cell layer. This behavior was observed throughout the growth of the superlattice, indicating a layer-by-layer growth mode.

The structure of the superlattices was characterized by $\mathrm{x}$-ray reflectivity (XRR), high-resolution x-ray diffraction (XRD), and Z-contrast scanning transmission electron

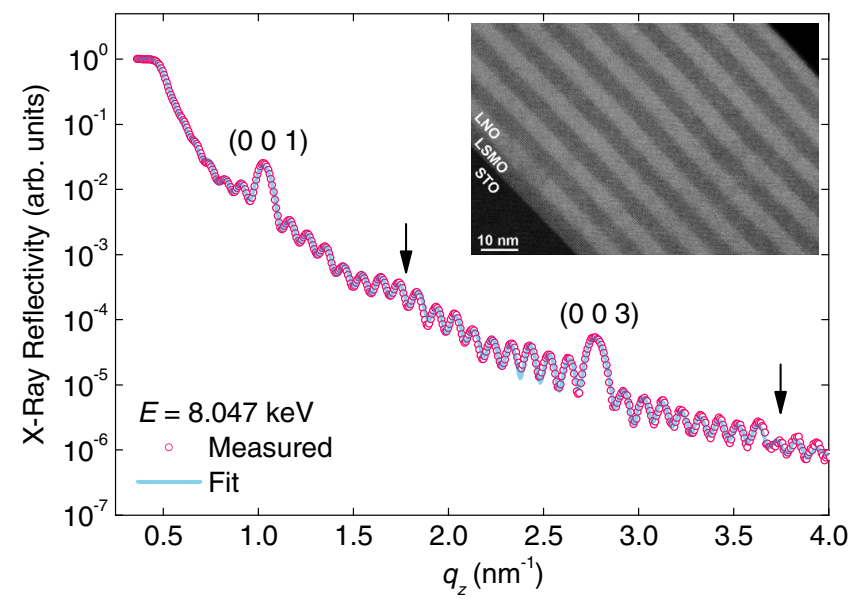

FIG. 1. Structural characterization of $\left(\mathrm{LaNiO}_{3}\right)_{n} /$ $\left(\mathrm{La}_{2 / 3} \mathrm{Sr}_{1 / 3} \mathrm{MnO}_{3}\right)_{9}$ superlattices. Measured x-ray reflectivity and fit for the $n=9$ superlattice on $\mathrm{SrTiO}_{3}$. The arrows mark the position of the even-order superlattice reflections, which are strongly suppressed by the structural symmetry of the sample. (Inset) High-resolution transmission electron micrograph of the same superlattice.

microscopy (STEM) measurements. Figure 1 shows XRR results for the $n=9$ superlattice, which are representative of all the samples studied here. From XRR data, we determine that the thickness of each superlattice period is within $0.5 \%$ of the designed value and that the $\mathrm{LNO} /$ LSMO interfacial roughness is less than one unit cell. Additional details about the growth and characterization may be found in the Ref. [40].

To probe the electronic structure and magnetism within the LNO spacer layer, we performed x-ray absorption spectroscopy (XAS) and x-ray magnetic circular dichroism (XMCD) measurements around the $\mathrm{Ni} L_{2}$ edge. Figure 2 shows the results for a series of LNO/LSMO superlattices with different $\mathrm{LNO}$ layer thicknesses, as well as $\mathrm{NiO}\left(\mathrm{Ni}^{2+}\right)$ and $\mathrm{LaNiO}_{3}$ (nominally $\mathrm{Ni}^{3+}$ ) reference spectra. The evolution of the $\mathrm{Ni} L_{2}$ peak shape and position shows an unambiguous change in the Ni valence state from nearly $\mathrm{Ni}^{3+}$ when $n=9$ to predominantly $\mathrm{Ni}^{2+}$ for $n=1$, with a characteristic double-peak line shape. This result is consistent with charge transfer confined to a few unit cells at the interface, in agreement with previous studies on manganite/nickelate interfaces [14,41]. The distinct doublepeak $\mathrm{Ni}^{2+} L_{2}$ line shape is in contrast to what is found in insulating ultrathin films of LNO, where a small, low-energy shoulder develops, similar to charge-ordered nickelates [42]. Complementary measurements at the $\mathrm{Mn} L_{2,3}$ edge are compatible with a mixture of $\mathrm{Mn}^{3+}$ and $\mathrm{Mn}^{4+}$ valence states, as expected for this composition of LSMO (see Fig. S4 of the Supplemental Materials [40]). While we do not observe any significant differences between the Mn XAS spectra for the samples measured here, signatures of a predominantly $\mathrm{Mn}^{4+}$ valence state were observed in previous work on $\mathrm{LaNiO}_{3} / \mathrm{LaMnO}_{3}$ superlattices with 2-unit-cell-thick 


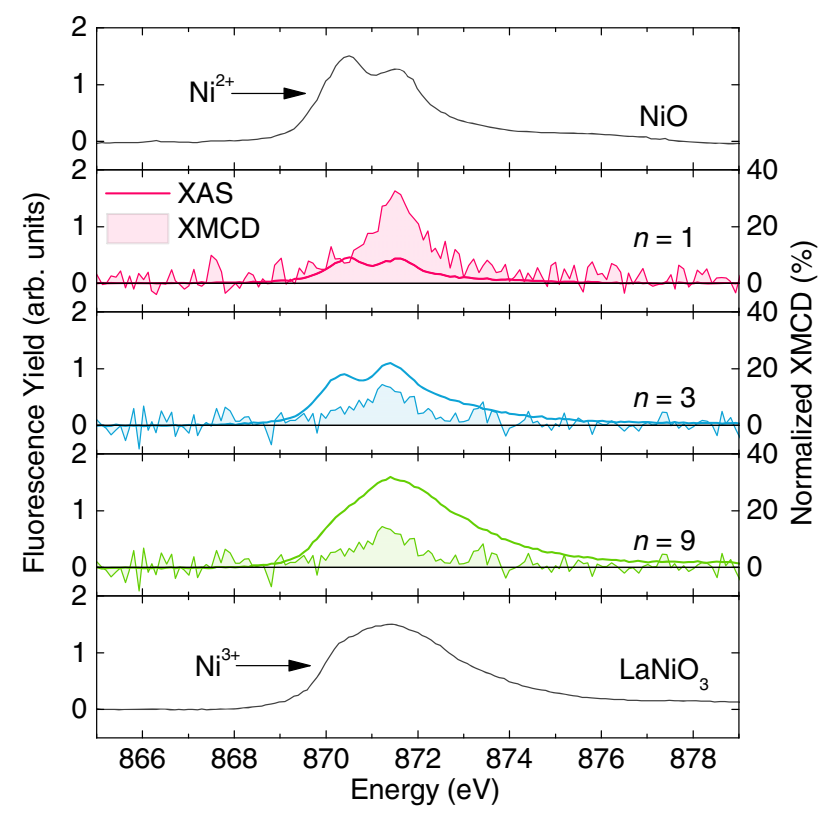

FIG. 2. Interfacial charge transfer and magnetic dichroism. X-ray absorption spectroscopy at the $\mathrm{Ni} L_{2}$ edge for $\left(\mathrm{LaNiO}_{3}\right)_{n} /$ $\left(\mathrm{La}_{2 / 3} \mathrm{Sr}_{1 / 3} \mathrm{MnO}_{3}\right)_{9}$ superlattices (thick lines) showing progression from $\mathrm{Ni}^{3+}$ to $\mathrm{Ni}^{2+}$ as $n$ is varied from 9 to 1 . The shaded regions show the $\mathrm{x}$-ray magnetic dichroism over the same energy range, confirming the existence of magnetism on the $\mathrm{Ni}$ sites.

$\mathrm{LaMnO}_{3}$ layers, consistent with a transfer of electrons from the manganite to the nickelate layer [14].

The XMCD spectra measured in an applied in-plane field of about $250 \mathrm{mT}$ at $110 \mathrm{~K}$ (shown as shaded regions in Fig. 2) confirm the presence of a net magnetic moment within the LNO layers. The shape of the XMCD spectra at the Ni $L_{2}$ peak for low- $n$ samples is very similar to that seen in the ferromagnetic double perovskite $\mathrm{La}_{2} \mathrm{MnNiO}_{6}$, where the $\mathrm{Ni}$ is in a $2+$ oxidation state $[43,44]$. We note that the total fluorescence-yield technique used here has a probe depth comparable to the sample thickness and that the XMCD spectra shown in Fig. 2 are not normalized by the volume of Ni probed, which changes as $n$ is varied. The magnetization of the LNO layers in the $n=3$ superlattice was investigated with $\mathrm{x}$-ray resonant magnetic scattering (XRMS) measurements, which are consistent with a commensurate modulation of the superlattice structure and the in-plane component of Ni magnetization that is parallel to the applied field (Fig. S5 of the Supplemental Materials [40]).

To further explore the magnetization profile of the superlattices, we carried out polarized neutron reflectometry (PNR) measurements using the polarized beam reflectometer at the Center for Neutron Research at the National Institute of Standards and Technology. The samples were cooled from room temperature in a 5-mT in-plane field applied along the [100] direction, which is parallel to $\mathbf{P}$, the polarization axis of the incident neutrons. PNR measurements of the superlattices grown on STO were carried out at temperatures above $110 \mathrm{~K}$, while superlattices grown on LSAT were investigated down to $10 \mathrm{~K}$ [45]. We are able to determine the depth profile of the magnitude and orientation of the in-plane magnetization within each layer by measuring both the non-spin-flip (NSF) reflectivities $\mathrm{R}^{\uparrow \uparrow}$ and $\mathrm{R}^{\downarrow \downarrow}$ and the spin-flip (SF) reflectivities $\mathrm{R}^{\uparrow \downarrow}$ and $\mathrm{R} \downarrow \uparrow$. Here, the superscripts denote the initial and final neutron spin states. Within the Born approximation, the NSF reflectivities depend on both $\rho(z)$ (the nuclear scattering length density) and $M_{\|}(z)$ (the projection of the in-plane magnetization parallel to the neutron polarization direction and applied magnetic field). Here, $z$ is the coordinate perpendicular to the plane of the film and interfaces. The SF reflectivities are only sensitive to $M_{\perp}(z)$, the projection of the magnetization that is perpendicular to the polarization direction and parallel to the interfaces.

The PNR measurements reveal a strongly modulated magnetization within the superlattices. Figures 3(a)-3(c) show the PNR spectra measured at $T=110 \mathrm{~K}$ in an applied field of $1.2 \pm 0.5 \mathrm{mT}$ as a function of $q_{z}$, the momentum transfer along the surface normal, for the superlattices with $n=3,6$, and 9 grown on STO. In all three samples, we observe splitting between the NSF reflectivities at the critical edge and at the superlattice Bragg reflections. This indicates a modulated profile for $M_{\|}(z)$ that is commensurate with the superlattice period.

Because of the symmetry of the $n=9$ superlattice, the chemical contribution to the even-order Bragg peaks is strongly suppressed, as evidenced by the XRR measurements (Fig. 1), further confirming the high structural quality of the superlattices. In the non-spin-flip PNR spectra shown in Fig. 3(c), however, we observe a pronounced (002) peak at $q_{z} \sim 1.8 \mathrm{~nm}^{-1}$, demonstrating that the magnetic profile does not follow the chemical structure exactly. To account for this observation, we have considered three scenarios for the interface magnetization: (i) an induced magnetization within the LNO at the interface, (ii) a reduced magnetization within the interfacial LSMO, and (iii) a combination of these two effects. Through detailed fitting using REFL1D [46], we find that an induced magnetization on the interfacial $\mathrm{Ni}$ sites alone is not sufficient to quantitatively explain the observed (002) peak. Rather, our fitting shows that the magnetization of the LSMO is reduced from its bulk value of about $550 \mathrm{kA} / \mathrm{m}$ (about $3.5 \mu_{\mathrm{B}} / \mathrm{Mn}$ ) to about $315 \mathrm{kA} / \mathrm{m}$ (about $2.0 \mu_{\mathrm{B}} / \mathrm{Mn}$ ) within 1-2 unit cells of the interface, in agreement with the length scale for interfacial charge transfer that we found in our XAS measurements (Fig. 2).

Furthermore, we see clear evidence for noncollinear alignment between the magnetization of adjacent LSMO layers. In the superlattices with $n=3$ and $n=9$, our measurements reveal an additional peak in both SF reflectivities at $q_{z} \sim 0.7 \mathrm{~nm}^{-1}(n=3)$ and $0.5 \mathrm{~nm}^{-1}(n=9)$, which correspond to the positions of the $\left(00 \frac{1}{2}\right)$ reflections. Thus, there exists a magnetization component $M_{\perp}(z)$ that 

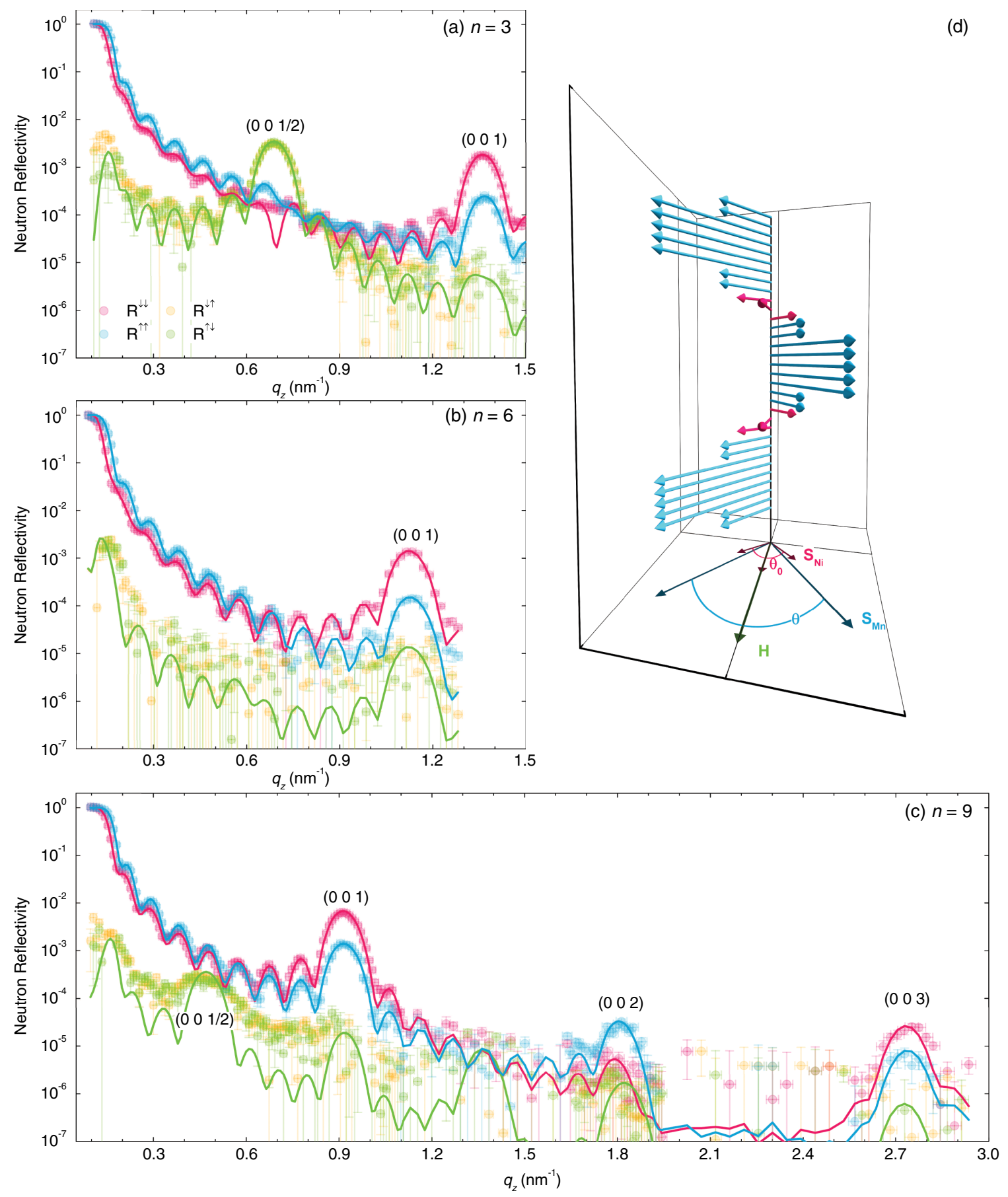

FIG. 3. Polarized neutron reflectometry for $\left(\mathrm{LaNiO}_{3}\right)_{n} /\left(\mathrm{La}_{2 / 3} \mathrm{Sr}_{1 / 3} \mathrm{MnO}_{3}\right)_{9}$ superlattices on $\mathrm{SrTiO}_{3}$. Measured (symbols) and fit (lines) PNR spectra for superlattices with $n=3$ (a), $n=6$ (b), and $n=9$ (c). All spectra were measured at $T=110 \mathrm{~K}$ with an applied in-plane field of $1.2 \mathrm{mT}$. (d) Schematic magnetic structure within the $n=3$ superlattice, where the LSMO and LNO layers are shown in blue and pink, respectively.

lies perpendicular to the applied field direction, which is modulated with twice the periodicity of the superlattice. The $\left(00 \frac{1}{2}\right)$ peak in the $n=9$ superlattice is weaker than in the $n=3$ superlattice, consistent with a smaller coupling angle in this sample. In the superlattice with $n=6$ [Fig. 3(b)], however, the $\left(00 \frac{1}{2}\right)$ peak is completely absent, indicating that adjacent LSMO layers are ferromagnetically aligned, even at the lowest measurement fields. In the $n=9$ superlattice, the $\left(00 \frac{1}{2}\right)$ peak is broadened relative to the (001) peak, which may indicate that $\theta$, the angle between the magnetization of adjacent LSMO layers, is not constant throughout the thickness of the superlattice. 


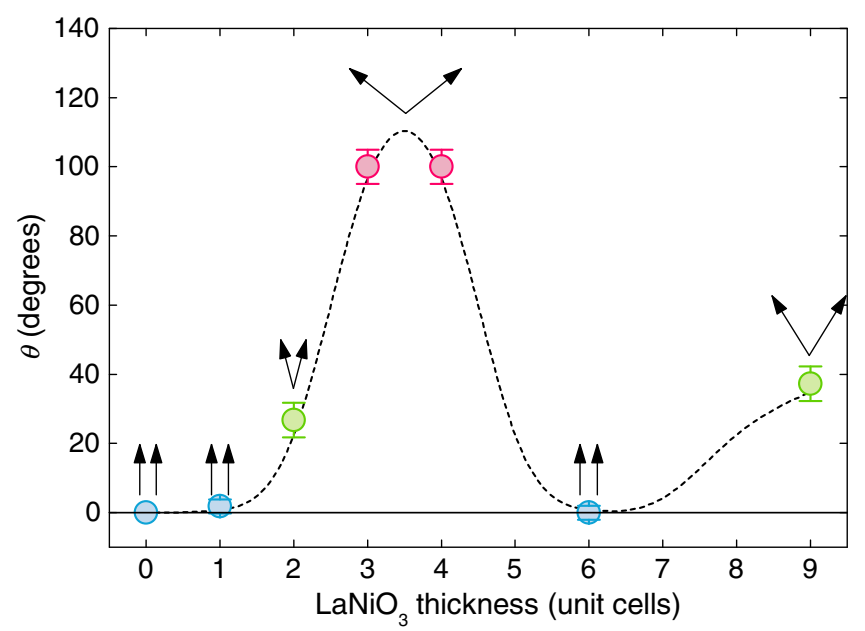

FIG. 4. Oscillatory noncollinear coupling in $\left(\mathrm{LaNiO}_{3}\right)_{n} /$ $\left(\mathrm{La}_{2 / 3} \mathrm{Sr}_{1 / 3} \mathrm{MnO}_{3}\right)_{9}$ superlattices. Dependence of coupling angle, $\theta$, on LNO thickness for superlattices grown on $\mathrm{SrTiO}_{3}$ at $T=110 \mathrm{~K}$ and $H=1.2 \mathrm{mT}$. The error bars are representative of the model-to-model variation in best-fit values of $\theta$ obtained for different chemical and magnetic profiles, which is much larger than the uncertainty in $\theta$ for a single model, as described in Ref. [40]. The line is a guide to the eye.

We have attempted fitting of the PNR data for each superlattice with a number of model structures, and we find that $\theta$ is insensitive $\left( \pm 5^{\circ}\right)$ to the details of the model used (see Fig. S3 of the Supplemental Materials [40] for additional details).

To study how the coupling angle varies with LNO thickness, we carried out PNR measurements on superlattices with $1 \leq n \leq 9$. Figure 4 shows how the value of $\theta$ obtained from fitting the PNR spectra shown in Fig. 3 changes as the thickness of LNO is varied. The coupling angle is found to oscillate with a period of about 5-7 unit cells. Remarkably, we find that the LSMO magnetization never attains complete antiferromagnetic $\left(180^{\circ}\right)$ alignment, as previously reported for $\mathrm{La}_{2 / 3} \mathrm{Ba}_{1 / 3} \mathrm{MnO}_{3} / \mathrm{LaNiO}_{3}$ multilayers [17-19]. Rather, the noncollinearity reaches a maximum of around $100^{\circ}$ for $n=3$ and 4 . This behavior is qualitatively different from that found for conventional metallic multilayers, such as $\mathrm{Fe} / \mathrm{Cr}$, where noncollinear magnetic alignments are typically only observed in a narrow regime of spacer layer thickness, where the interlayer exchange coupling transitions between collinear ferromagnetic and antiferromagnetic alignments [47]. The behavior shown in Fig. 4 is also remarkably different from that recently reported for (111)-oriented $\mathrm{LaMnO}_{3} / \mathrm{LaNiO}_{3}$ superlattices, where noncollinear magnetism was only observed for a LNO thickness of exactly 7 unit cells [16].

To uncover the origin of the surprising behavior shown in Fig. 4, we examine two possible models for the interlayer exchange coupling [48]. We first consider a phenomenological description based on bilinear (collinear) and biquadratic (noncollinear) effects, which is widely used to model the exchange interactions within conventional metallic multilayers [24-26]:

$$
F_{\mathrm{BLBQ}}(\theta)=-J_{\mathrm{BL}} \cos (\theta)-J_{\mathrm{BQ}} \cos ^{2}(\theta) .
$$

The bilinear term $J_{\mathrm{BL}}$ derives from the magnetism in the LNO spacer layer and oscillates in sign with LNO thickness, favoring either parallel or antiparallel alignment of the LSMO layers. $J_{\mathrm{BQ}}$ is usually attributed to defects such as interfacial roughness, steps, or magnetic impurities in the spacer layer, and it favors a $90^{\circ}$ alignment for $J_{\mathrm{BQ}}<0$. In principle, an additional term due to anisotropy may be present. However, as described in Ref. [40], SQUID magnetometry measurements confirm that the in-plane magnetocrystalline anisotropy in our samples is relatively weak $\left(\left|K t_{\mathrm{LSMO}}\right| \lesssim 10^{-6} \mathrm{Jm}^{-2} \ll\left|J_{\mathrm{BL}}\right| \sim\left|J_{\mathrm{BQ}}\right| \approx 10^{-5}-10^{-4} \mathrm{~J} \mathrm{~m}^{-2}\right)$ and may be ignored. Furthermore, neutron scattering measurements on the $n=3$ superlattice grown on LSAT with the magnetic field applied along the $\langle 110\rangle$ direction yields the same value of $\theta$ as for measurements with the field applied along $\langle 100\rangle$. In the absence of anisotropy, the value of $\theta$ at $H=0$ is determined by the ratio of $J_{\mathrm{BL}}$ to $J_{\mathrm{BQ}}$, favoring noncollinear alignment for $\left|J_{\mathrm{BQ}}\right|>\left|J_{\mathrm{BL}}\right| / 2$.

As an alternative mechanism to explain the observed noncollinear exchange coupling, we propose the formation of a spin-helix within the LNO layers. Helimagnetic states should arise generally for large, localized spins and a magnetic susceptibility $\chi(q)$ with peaks that favor a spatially oscillating magnetic order [49]. Transfer of electrons from the LSMO into the LNO layer creates interfacial $\mathrm{Ni}^{2+}$ states, as shown by the XAS measurements in Fig. 2. While the underlying electronic structure of these $\mathrm{Ni}^{2+}$ states is presently unclear, they are expected to form localized moments with $S=1$, within a region of about 2 unit cells of each interface. Photoemission measurements and first-principles calculations indicate that the Fermi surface of metallic nickelates with $\mathrm{Ni}^{3+}$ has a nested character [18,50,51]. For realistic hopping amplitudes, calculations of $\chi(q)$ for these materials show a peak between $2 \pi(0.15,0,0)$ and $2 \pi(0.18,0,0)$, corresponding to a period of about 5-7 unit cells along the (001) direction [52,53]. This happens to be in agreement with the oscillation period that we observe in Fig. 4, despite differences between the electronic and magnetic properties of the LNO layers studied here and those found in bulklike rare-earth nickelates. A full theoretical description of helimagnetism in the LNO layer in our samples would need to include the localized $\mathrm{Ni}^{2+}$ spins near the interface, magnetic instabilities in the spatially varying electronic structure, and the interfacial Ni-Mn exchange interaction in a self-consistent manner [54], which is beyond the scope of this work.

In many magnetic systems, noncollinear magnetism arises from the Dzyaloshinsky-Moriya (D-M) interaction. However, based on the symmetry and structural properties of the superlattices, we do not believe that this mechanism 
can be the primary origin of the helical magnetic state postulated for the LNO layers. Furthermore, interfacial electric fields that lead to a D-M interaction with the D-M vector perpendicular to the interface do not explain the observed alternation of the chirality of the spin helixes in LNO [31]. Cooling through $T_{C}$ in a magnetic field, however, as we do here, tends to preferentially align the magnetization of the LSMO layers with the applied field, leading to a natural alternation of helicity in the nickelate layers [Fig. 3(d)]. A detailed discussion of the role of the D-M interaction in these superlattices is contained in Ref. [40].

We now construct an effective energy function for the spin-helix model, supposing that the amplitudes of the magnetization in the manganite and nickelate layers are $S_{\mathrm{Mn}}$ and $S_{\mathrm{Ni}}$, and that the helix rotates by angle $\theta_{0}$ from one manganite layer to the neighboring one, as shown schematically in Fig. 3(d). If we assume that intrahelix stiffness is significantly higher than the coupling to the manganite layers, the interfacial exchange energy in the zero magnetic field is

$$
F_{\mathrm{S}-\mathrm{H}}(\theta)=-2 J S_{\mathrm{Mn}} S_{\mathrm{Ni}} \cos \left(\frac{\theta-\theta_{0}}{2}\right),
$$

where $J$ is the exchange coupling between LSMO and LNO. In a magnetic field, we need to include the Zeeman energy of both the $\mathrm{Mn}$ and $\mathrm{Ni}$ atoms. The former is simply $-g \mu_{\mathrm{B}} S_{\mathrm{Mn}} t H \cos (\theta / 2)$, with $t$ the thickness of the LSMO in unit cells, while the latter has to be summed over all of the
$\mathrm{Ni}$ atoms in the helix. For a rigid $\mathrm{Ni}$ spin helix, we can ignore the Zeeman contribution from the $\mathrm{Ni}$ spins, and the magnetic-field dependence of the coupling angle may be determined exactly through minimization of Eq. (2) in the presence of a magnetic field. It is given by

$$
\theta(H)=2 \tan ^{-1}\left[\frac{\sin \left(\frac{\theta_{0}}{2}\right)}{\cos \left(\frac{\theta_{0}}{2}\right)+\frac{g \mu_{\mathrm{B}} t H}{2 J S_{\mathrm{Ni}}}}\right] .
$$

To distinguish between the bilinear-biquadratic and spinhelix models, we measure the coupling angle as a function of an in-plane magnetic field and temperatures as low as $10 \mathrm{~K}$. The results for an $n=3$ superlattice grown on LSAT and measured at $T=125 \mathrm{~K}$ are shown in Fig. 5(a). For this sample, the magnetization of neighboring LSMO layers approaches ferromagnetic alignment at around $600 \mathrm{mT}$, above which no $\left(00 \frac{1}{2}\right)$ spin-flip peak is measurable. We carry out least-squares fitting to Eq. (3), and the resulting fit is shown by the solid line in Fig. 5(a). Taking $S_{\mathrm{Ni}}=1$, we obtain an exchange coupling between $\mathrm{Mn}$ and $\mathrm{Ni}$ of $J=3.4 \times 10^{-5} \mathrm{~J} \mathrm{~m}^{-2}(J=32 \mu \mathrm{eV}$ per interface unit cell $)$, much less than the value predicted for $J_{\mathrm{Mn}-\mathrm{Ni}}$ at the (001) $\mathrm{LaNiO}_{3} / \mathrm{LaMnO}_{3}$ interface [55]. In exchange bias systems, comparable differences between the predicted and measured interfacial exchange coupling energy are observed, and their origin remains an open question [56]. Despite the simplicity of this model, it captures two important characteristics of the data: (i) the sharp initial drop in coupling angle at low fields, and (ii) the asymptotic approach to alignment at high fields [57].
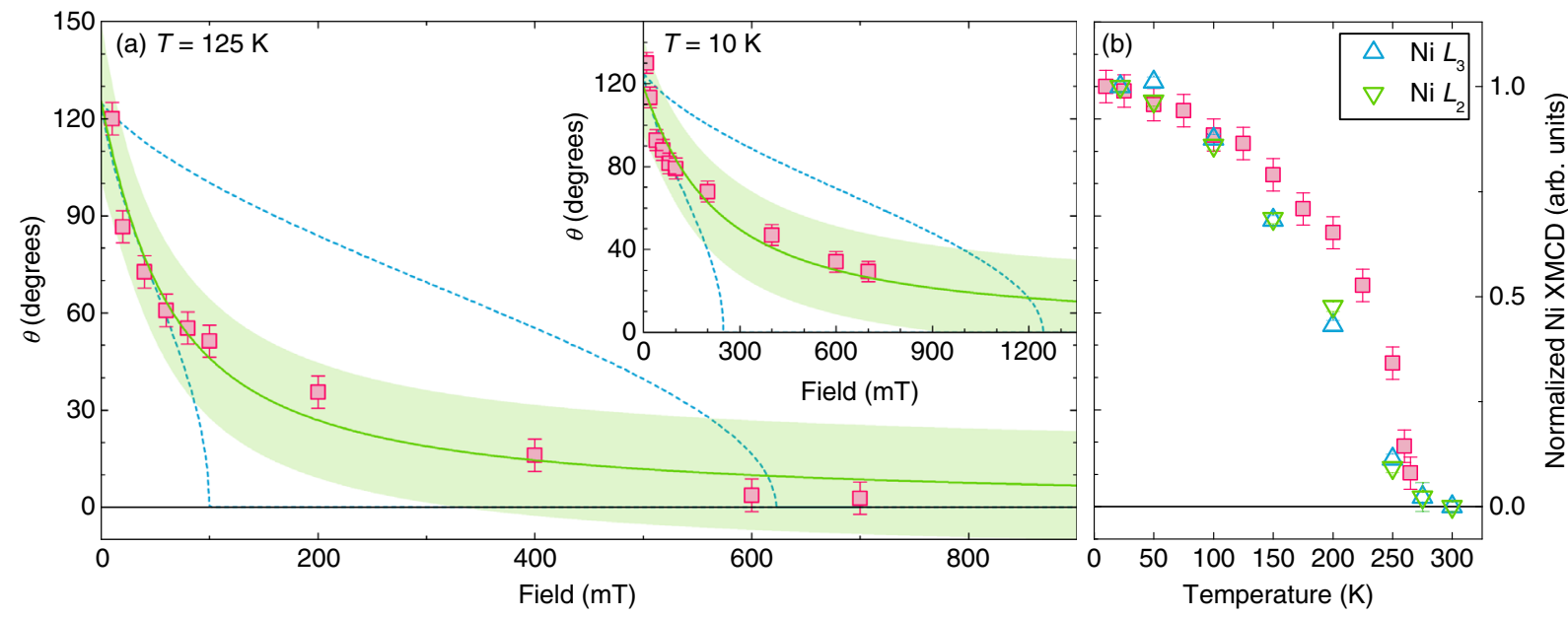

FIG. 5. Magnetic-field and temperature dependence of the coupling angle for the $\left[\left(\mathrm{LaNiO}_{3}\right)_{3} /\left(\mathrm{La}_{2 / 3} \mathrm{Sr}_{1 / 3} \mathrm{MnO}_{3}\right)_{9}\right]_{14}$ superlattice on LSAT. (a) Evolution of the coupling angle with an in-plane field applied along the (100) direction at $125 \mathrm{~K}$ (a) and $10 \mathrm{~K}$ (inset). The solid line shows the best fit to Eq. (3) with $J=3.4 \times 10^{-5} \mathrm{~J} \mathrm{~m}^{-2}$ at $125 \mathrm{~K}$ and $J=1.2 \times 10^{-4} \mathrm{~J} \mathrm{~m}^{-2}$ at $10 \mathrm{~K}$, assuming $S_{\mathrm{Ni}}=1$ at both temperatures. Shaded regions represent the $95 \%$ prediction interval using Eq. (3). Dashed lines show the expected behavior within the bilinear-biquadratic model [Eq. (1)] for $J_{\mathrm{BL}}=-1.00 \times 10^{-4} \mathrm{~J} \mathrm{~m}^{-2}$ and $J_{\mathrm{BQ}}=-8.75 \times 10^{-5} \mathrm{~J} \mathrm{~m}^{-2}$ (upper curve, main panel), $J_{\mathrm{BL}}=-1.60 \times 10^{-5} \mathrm{~J} \mathrm{~m}^{-2}$ and $J_{\mathrm{BQ}}=-1.40 \times 10^{-5} \mathrm{~J} \mathrm{~m}^{-2}$ (lower curve, main panel), $J_{\mathrm{BL}}=-2.00 \times 10^{-4} \mathrm{~J} \mathrm{~m}^{-2}$ and $J_{\mathrm{BQ}}=-1.75 \times 10^{-4} \mathrm{~J} \mathrm{~m}^{-2}$ (upper curve, inset), and $J_{\mathrm{BL}}=-4.00 \times 10^{-5} \mathrm{~J} \mathrm{~m}^{-2}$ and $J_{\mathrm{BQ}}=-3.00 \times 10^{-5} \mathrm{~J} \mathrm{~m}^{-2}$ (lower curve, inset). (b) Variation of the coupling angle with temperature in an applied field of $1.2 \mathrm{mT}$. Error bars $\left( \pm 5^{\circ}\right)$ indicate the estimated uncertainty in fitting of the PNR spectra, and they are greater than the errors that arise from counting statistics. 
On the other hand, the response calculated within the bilinear-biquadratic model (dashed lines) according to Eq. (1) does not agree with the measured field dependence. Here, we have fixed the ratio $J_{\mathrm{BL}} / J_{\mathrm{BQ}}$ to agree with the measured value of $\theta$ at $H<1.2 \mathrm{mT}$, while the magnitudes of $J_{\mathrm{BL}}$ and $J_{\mathrm{BQ}}$ were chosen to match either the low-field behavior or the ferromagnetic alignment field. While the selected values of $J_{\mathrm{BL}}$ and $J_{\mathrm{BQ}}$ are comparable to those previously reported in a number of conventional metallic $[47,58,59]$ and oxide [60] systems, we find that they are unable to reproduce the observed field dependence of the coupling angle. For example, to reproduce the measured alignment field of about $600 \mathrm{mT}$ requires values of $J_{\mathrm{BL}}$ and $J_{\mathrm{BQ}}$ that lead to large discrepancies between predicted and observed behavior at lower fields. Furthermore, choosing $J_{\mathrm{BL}}$ and $J_{\mathrm{BQ}}$ to give the correct low-field behavior results in a much lower alignment field of only $125 \mathrm{mT}$. Additional measurements carried out on the same sample at $T=10 \mathrm{~K}$ [Fig. 5 (inset)] demonstrate that the noncollinearity persists to above $700 \mathrm{mT}$ at lower temperatures, the largest field that we are able to apply within the PNR apparatus.

The temperature dependence of the exchange coupling is also unlike that observed in conventional metallic heterostructures, where noncollinear behavior is often restricted to a limited range of temperatures $[59,61]$. For the $n=3$ superlattice on LSAT, we find that the noncollinearity persists to about $265 \mathrm{~K}$ [Fig. 5(b)], close to the onset temperature of magnetization in the LNO layers found with XMCD (see Ref. [40]). This is above the highest reported magnetic ordering temperature for the rare-earth nickelates of about $250 \mathrm{~K}$ for $\mathrm{Nd}_{1-x} \mathrm{Sm}_{x} \mathrm{NiO}_{3}$ [62], and it highlights the intimate connection between charge transfer, local $\mathrm{Ni}$ moments, and noncollinear magnetic ordering.

In conclusion, we have demonstrated that interfacial charge transfer drives an oscillatory noncollinear magnetic coupling between ferromagnetic $\mathrm{La}_{2 / 3} \mathrm{Sr}_{1 / 3} \mathrm{MnO}_{3}$ layers separated by thin $\mathrm{LaNiO}_{3}$ spacers. The measured field and temperature dependence of the noncollinearity prove that a new mechanism, which cannot be explained by a combination of conventional bilinear and biquadratic interactions, is responsible for the interlayer exchange coupling in this system. To explain the observed behavior, we propose the formation of a helical magnetic state within LNO. Such a helimagnetic state may arise from the cooperative interaction between localized $\mathrm{Ni}^{2+}$ states that result from interfacial charge transfer and a magnetic instability that is ubiquitous in the rare-earth nickelates. This mechanism does not require strong spin-orbit coupling or D-M interactions. Direct measurements of the magnetic structure of the nickelate layers, using resonant $\mathrm{x}$-ray scattering for example, are needed to verify our proposal.

Charge transfer and interfacial electronic reconstructions play a critical role in the creation of novel collective phases, not only at interfaces between insulating materials but, as we show here, also metallic oxide interfaces. This phenomenon is expected to be broadly applicable to metallic oxides that are derived from Mott insulators. Furthermore, we envisage applications where electric fields may be used to control the charge transfer, and thus the electronic and magnetic structure, near interfaces between correlated metallic oxides.

\section{ACKNOWLEDGMENTS}

We are grateful to Y. H. Liu, M. D. Stiles, J. A. Borchers, and B.B. Maranville for valuable discussions. Work at Argonne National Laboratory, including the use of the Center for Nanoscale Materials and Advanced Photon Source, was supported by the U.S. Department of Energy, Office of Basic Energy Sciences under Contract No. DEAC02-06CH11357. J. D.H, O. G. H., I. M., S. G. E.t.V, and A. B. acknowledge support from the U.S. Department of Energy, Office of Basic Energy Science, Materials Science and Engineering Division. We acknowledge the support of the National Institute of Standards and Technology, U.S. Department of Commerce, in providing the neutron research facilities used in this work. J. K. and J. M. Z. are supported as part of the Center for Emergent Superconductivity, an Energy Frontier Research Center funded by the U.S. Department of Energy, Office of Science, Office of Basic Energy Sciences, under Grant No. DE-AC02-98CH10886. Work by G. F., D. M., and M. P. M. D. is supported by U.S. Department of Energy, Office of Basic Energy Sciences, Early Career Award Program under Grant No. 1047478. Experiments were in part performed at the REIXS beamline of the Canadian Light Source, which is funded by the Canada Foundation for Innovation, the Natural Sciences and Engineering Research Council of Canada, National Research Council Canada, and the Canadian Institute of Health Research, the Government of Saskatchewan, WD Canada, and the University of Saskatchewan. The Diamond Light Source is acknowledged for beam time allocated on I10 under Proposal Reference No. SI-9626.

[1] H. Y. Hwang, Y. Iwasa, M. Kawasaki, B. Keimer, N. Nagaosa, and Y. Tokura, Emergent Phenomena at Oxide Interfaces, Nat. Mater. 11, 103 (2012).

[2] J. Junquera and P. Ghosez, Critical Thickness for Ferroelectricity in Perovskite Ultrathin Films, Nature (London) 422, 506 (2003).

[3] M. Y. Zhuravlev, R. F. Sabirianov, S. S. Jaswal, and E. Y. Tsymbal, Giant Electroresistance in Ferroelectric Tunnel Junctions, Phys. Rev. Lett. 94, 246802 (2005).

[4] J. D. Burton and E. Y. Tsymbal, Evolution of the Band Alignment at Polar Oxide Interfaces, Phys. Rev. B 82, 161407 (2010).

[5] P. A. Salvador, A.-M. Haghiri-Gosnet, B. Mercey, M. Hervieu, and B. Raveau, Growth and Magnetoresistive Properties of $\left(\mathrm{LaMnO}_{3}\right)_{m}\left(\mathrm{SrMnO}_{3}\right)_{n}$ Superlattices, Appl. Phys. Lett. 75, 2638 (1999). 
[6] A. Ohtomo, D. A. Muller, J. L. Grazul, and H. Y. Hwang, Artificial Charge-Modulation in Atomic-Scale Perovskite Titanate Superlattices, Nature (London) 419, 378 (2002).

[7] A. Ohtomo and H. Y. Hwang, A High-Mobility Electron Gas at the $\mathrm{LaAlO}_{3} / \mathrm{SrTiO}_{3}$ Heterointerface, Nature (London) 427, 423 (2004).

[8] Y. Hotta, T. Susaki, and H. Y. Hwang, Polar Discontinuity Doping of the $\mathrm{LaVO}_{3} / \mathrm{SrTiO}_{3}$ Interface, Phys. Rev. Lett. 99, 236805 (2007).

[9] J. Liu, S. Okamoto, M. van Veenendaal, M. Kareev, B. Gray, P. Ryan, J. W. Freeland, and J. Chakhalian, Quantum Confinement of Mott Electrons in Ultrathin $\mathrm{LaNiO}_{3} /$ $\mathrm{LaAlO}_{3}$ Superlattices, Phys. Rev. B 83, 161102 (2011).

[10] A. V. Boris et al., Dimensionality Control of Electronic Phase Transitions in Nickel-Oxide Superlattices, Science 332, 937 (2011).

[11] M. Gibert, P. Zubko, R. Scherwitzl, J. Iñiguez, and J.-M. Triscone, Exchange Bias in $\mathrm{LaNiO}_{3}-\mathrm{LaMnO}_{3}$ Superlattices, Nat. Mater. 11, 195 (2012).

[12] Y. Liu, F. A. Cuellar, Z. Sefrioui, J. W. Freeland, M. R. Fitzsimmons, C. Leon, J. Santamaria, and S. G. E. te Velthuis, Emergent Spin Filter at the Interface between Ferromagnetic and Insulating Layered Oxides, Phys. Rev. Lett. 111, 247203 (2013).

[13] A. Frano et al., Orbital Control of Noncollinear Magnetic Order in Nickel Oxide Heterostructures, Phys. Rev. Lett. 111, 106804 (2013).

[14] J. Hoffman, I. C. Tung, B. B. Nelson-Cheeseman, M. Liu, J. W. Freeland, and A. Bhattacharya, Charge Transfer and Interfacial Magnetism in $\left(\mathrm{LaNiO}_{3}\right)_{n} /\left(\mathrm{LaMnO}_{3}\right)_{2}$ Superlattices, Phys. Rev. B 88, 144411 (2013).

[15] F. A. Cuellar et al., Reversible Electric-Field Control of Magnetization at Oxide Interfaces, Nat. Commun. 5, 4215 (2014).

[16] M. Gibert, M. Viret, P. Zubko, N. Jaouen, J.-M. Tonnerre, A. Torres-Pardo, S. Catalano, A. Gloter, O. Stéphan, and J.-M. Triscone, Interlayer Coupling through a DimensionalityInduced Magnetic State, Nat. Commun. 7, 11227 (2016).

[17] K. R. Nikolaev, A. Bhattacharya, P. A. Kraus, V. A. Vas'ko, W. K. Cooley, and A. M. Goldman, Indications of Antiferromagnetic Interlayer Coupling in $\mathrm{La}_{2 / 3} \mathrm{Ba}_{1 / 3} \mathrm{MnO}_{3} /$ $\mathrm{LaNiO}_{3}$ Multilayers, Appl. Phys. Lett. 75, 118 (1999).

[18] K. R. Nikolaev, A. Y. Dobin, I. N. Krivorotov, W. K. Cooley, A. Bhattacharya, A. L. Kobrinskii, L. I. Glazman, R. M. Wentzovitch, E. D. Dahlberg, and A. M. Goldman, Oscillatory Exchange Coupling and Positive Magnetoresistance in Epitaxial Oxide Heterostructures, Phys. Rev. Lett. 85, 3728 (2000).

[19] I. N. Krivorotov, K. R. Nikolaev, A. Y. Dobin, A. M. Goldman, and E. D. Dahlberg, Exchange Field Induced Magnetoresistance in Colossal Magnetoresistance Manganites, Phys. Rev. Lett. 86, 5779 (2001).

[20] M. N. Baibich, J. M. Broto, A. Fert, F. Nguyen Van Dau, F. Petroff, P. Etienne, G. Creuzet, A. Friederich, and J. Chazelas, Giant Magnetoresistance of $(001) \mathrm{Fe} /(001) \mathrm{Cr}$ Magnetic Superlattices, Phys. Rev. Lett. 61, 2472 (1988).

[21] G. Binasch, P. Grünberg, F. Saurenbach, and W. Zinn, Enhanced Magnetoresistance in Layered Magnetic Structures with Antiferromagnetic Interlayer Exchange, Phys. Rev. B 39, 4828 (1989).
[22] S. S. P. Parkin, N. More, and K. P. Roche, Oscillations in Exchange Coupling and Magnetoresistance in Metallic Superlattice Structures: $\mathrm{Co} / \mathrm{Ru}, \mathrm{Co} / \mathrm{Cr}$, and $\mathrm{Fe} / \mathrm{Cr}$, Phys. Rev. Lett. 64, 2304 (1990).

[23] M. D. Stiles, Exchange Coupling in Magnetic Heterostructures, Phys. Rev. B 48, 7238 (1993).

[24] J. C. Slonczewski, Overview of Interlayer Exchange Theory, J. Magn. Magn. Mater. 150, 13 (1995).

[25] S. O. Demokritov, Biquadratic Interlayer Coupling in Layered Magnetic Systems, J. Phys. D 31, 925 (1998).

[26] M. D. Stiles, Interlayer Exchange Coupling, J. Magn. Magn. Mater. 200, 322 (1999).

[27] T. S. Santos, B. J. Kirby, S. Kumar, S. J. May, J. A. Borchers, B. B. Maranville, J. Zarestky, S. G. E. te Velthuis, J. van den Brink, and A. Bhattacharya, Delta Doping of Ferromagnetism in Antiferromagnetic Manganite Superlattices, Phys. Rev. Lett. 107, 167202 (2011).

[28] A. Brataas, G. Bauer, and P. Kelly, Non-collinear Magnetoelectronics, Phys. Rep. 427, 157 (2006).

[29] F. S. Bergeret, A. F. Volkov, and K. B. Efetov, Odd Triplet Superconductivity and Related Phenomena in Superconductor-Ferromagnet Structures, Rev. Mod. Phys. 77, 1321 (2005).

[30] S.-W. Cheong and M. Mostovoy, Multiferroics: A Magnetic Twist for Ferroelectricity, Nat. Mater. 6, 13 (2007).

[31] Y. Tokura and S. Seki, Multiferroics with Spiral Spin Orders, Adv. Mater. 22, 1554 (2010).

[32] Y. Tokura, S. Seki, and N. Nagaosa, Multiferroics of Spin Origin, Rep. Prog. Phys. 77, 076501 (2014).

[33] N. Nagaosa and Y. Tokura, Topological Properties and Dynamics of Magnetic Skyrmions, Nat. Nanotechnol. 8, 899 (2013).

[34] S. Banerjee, J. Rowland, O. Erten, and M. Randeria, Enhanced Stability of Skyrmions in Two-Dimensional Chiral Magnets with Rashba Spin-Orbit Coupling, Phys. Rev. X 4, 031045 (2014).

[35] J. Son, P. Moetakef, J. M. LeBeau, D. Ouellette, L. Balents, S. J. Allen, and S. Stemmer, Low-Dimensional Mott Material: Transport in Ultrathin Epitaxial $\mathrm{LaNiO}_{3}$ Films, Appl. Phys. Lett. 96, 062114 (2010).

[36] R. Scherwitzl, S. Gariglio, M. Gabay, P. Zubko, M. Gibert, and J.-M. Triscone, Metal-Insulator Transition in Ultrathin $\mathrm{LaNiO}_{3}$ Films, Phys. Rev. Lett. 106, 246403 (2011).

[37] J. Chakhalian et al., Asymmetric Orbital-Lattice Interactions in Ultrathin Correlated Oxide Films, Phys. Rev. Lett. 107, 116805 (2011).

[38] P. D. C. King, H. I. Wei, Y. F. Nie, M. Uchida, C. Adamo, S. Zhu, X. He, I. Božović, D. G. Schlom, and K. M. Shen, Atomic-Scale Control of Competing Electronic Phases in Ultrathin $\mathrm{LaNiO}_{3}$, Nat. Nanotechnol. 9, 443 (2014).

[39] D. P. Kumah, A. S. Disa, J. H. Ngai, H. Chen, A. Malashevich, J. W. Reiner, S. Ismail-Beigi, F. J. Walker, and C. H. Ahn, Tuning the Structure of Nickelates to Achieve Two-Dimensional Electron Conduction, Adv. Mater. 26, 1935 (2014).

[40] See Supplemental Material at http://link.aps.org/ supplemental/10.1103/PhysRevX.6.041038 for additional characterization of the structural, electronic, and magnetic properties of the $\mathrm{LaNiO}_{3} / \mathrm{La}_{2 / 3} \mathrm{Sr}_{1 / 3} \mathrm{MnO}_{3}$ superlattices. 
[41] J. C. Rojas Sánchez, B. Nelson-Cheeseman, M. Granada, E. Arenholz, and L. B. Steren, Exchange-Bias Effect at $\mathrm{La}_{0.75} \mathrm{Sr}_{0.25} \mathrm{MnO}_{3} / \mathrm{LaNiO}_{3}$ Interfaces, Phys. Rev. B 85, 094427 (2012).

[42] E. Sakai, M. Tamamitsu, K. Yoshimatsu, S. Okamoto, K. Horiba, M. Oshima, and H. Kumigashira, Gradual Localization of Ni $3 d$ States in $\mathrm{LaNiO}_{3}$ Ultrathin Films Induced by Dimensional Crossover, Phys. Rev. B 87, 075132 (2013).

[43] J. Blasco, J. García, M. C. Sánchez, J. Campo, G. Subías, and J. Pérez-Cacho, Magnetic Properties of $\mathrm{LaNi}_{1-x} \mathrm{Mn}_{x} \mathrm{O}_{3+\delta}$ Perovskites, Eur. Phys. J. B 30, 469 (2002).

[44] H. Guo, A. Gupta, M. Varela, S. J. Pennycook, and J. Zhang, Local Valence and Magnetic Characteristics of $\mathrm{La}_{2} \mathrm{NiMnO}_{6}$, Phys. Rev. B 79, 172402 (2009).

[45] Neutron scattering shows a threefold increase in mosaic spread below the cubic-to-tetragonal structural transition in STO, which is known to occur at about $105 \mathrm{~K}$.

[46] B. J. Kirby, P. A. Kienzle, B. B. Maranville, N. F. Berk, J. Krycka, F. Heinrich, and C. F. Majkrzak, Phase-Sensitive Specular Neutron Reflectometry for Imaging the Nanometer Scale Composition Depth Profile of Thin-Film Materials, Curr. Opin. Colloid Interface Sci. 17, 44 (2012).

[47] M. Rührig, R. Schäfer, A. Hubert, R. Mosler, J. A. Wolf, S. O. Demokritov, and P. Grünberg, Domain Observations on $\mathrm{Fe}-\mathrm{Cr}$-Fe Layered Structures. Evidence for a Biquadratic Coupling Effect, Phys. Status Solidi A 125, 635 (1991).

[48] Slonczewski has proposed a third mechanism - the proximity magnetism model-to explain noncollinear interlayer coupling in systems with antiferromagnetic spacer layers, such as $\mathrm{Cr}$ and Mn [24]. Such a model does not explain the observed variation in exchange coupling with spacer layer thickness, which is never antiferromagnetic. Furthermore, we have not found evidence for A-type or G-type antiferromagnetic ordering within the LNO layers in neutron diffraction experiments.

[49] Daniel I. Khomskii, Basic Aspects of the Quantum Theory of Solids: Order and Elementary Excitations (Cambridge University Press, New York, 2010).

[50] R. Eguchi, A. Chainani, M. Taguchi, M. Matsunami, Y. Ishida, K. Horiba, Y. Senba, H. Ohashi, and S. Shin, Fermi Surfaces, Electron-Hole Asymmetry, and Correlation Kink in a Three-dimensional Fermi Liquid $\mathrm{LaNiO}_{3}$, Phys. Rev. B 79, 115122 (2009).

[51] G. Fabbris et al., Orbital Engineering in Nickelate Heterostructures Driven by Anisotropic Oxygen Hybridization rather than Orbital Energy Levels, Phys. Rev. Lett. 117, 147401 (2016).
[52] S. B. Lee, R. Chen, and L. Balents, Metal-Insulator Transition in a Two-band Model for the Perovskite Nickelates, Phys. Rev. B 84, 165119 (2011).

[53] S. B. Lee, R. Chen, and L. Balents, Landau Theory of Charge and Spin Ordering in the Nickelates, Phys. Rev. Lett. 106, 016405 (2011).

[54] Y. Feng et al., Incommensurate Antiferromagnetism in a Pure Spin System via Cooperative Organization of Local and Itinerant Moments, Proc. Natl. Acad. Sci. USA 110, 3287 (2013).

[55] A. T. Lee and M. J. Han, Charge Transfer, Confinement, and Ferromagnetism in $\mathrm{LaMnO}_{3} / \mathrm{LaNiO}_{3}$ (001) Superlattices, Phys. Rev. B 88, 035126 (2013).

[56] J. Nogués and I. K. Schuller, Exchange Bias, J. Magn. Magn. Mater. 192, 203 (1999).

[57] For the $n=3$ superlattice on LSAT, the magnetization of the LSMO layers is not symmetric about the nominal field direction at the lowest measurement fields, unlike the samples prepared on $\mathrm{SrTiO}_{3}$. This may result from differences in epitaxial strain imposed by the two substrates. Despite this behavior at low fields, we still find that the angle between the magnetization of adjacent LSMO layers is well described by Eq. (3), which is also in agreement with the field dependence measured on a second sample on $\mathrm{SrTiO}_{3}$.

[58] A. Schreyer, J. F. Ankner, Th. Zeidler, H. Zabel, M. Schäfer, J. A. Wolf, P. Grünberg, and C. F. Majkrzak, Noncollinear and Collinear Magnetic Structures in Exchange Coupled Fe/Cr (001) Superlattices, Phys. Rev. B 52, 16066 (1995).

[59] E. E. Fullerton and S. D. Bader, Temperature-Dependent Biquadratic Coupling in Antiferromagnetically Coupled Fe/FeSi Multilayers, Phys. Rev. B 53, 5112 (1996).

[60] J. Park, D. R. Lee, Y. Choi, J. W. Freeland, K. .B. Lee, S. K. Sihna, K. R. Nikolaev, and A. M. Goldman, Quantifying Interlayer Exchange Coupling via Layer-Resolved Hysteresis Loops in Antiferromagnetically Coupled Manganitel Nickelate Superlattices, Appl. Phys. Lett. 95, 102504 (2009).

[61] D. Haskel, G. Srajer, Y. Choi, D. R. Lee, J. C. Lang, J. Meersschaut, J.S. Jiang, and S. D. Bader, Nature of Inhomogeneous Magnetic State in Artificial Fe/Gd Ferrimagnetic Multilayers, Phys. Rev. B 67, 180406 (2003).

[62] J. B. Torrance, P. Lacorre, A. I. Nazzal, E. J Ansaldo, and C. Niedermayer, Systematic Study of Insulator-Metal Transitions in Perovskites $\mathrm{RNiO}_{3}$, Phys. Rev. B 45, 8209 (1992). 